\title{
THE USE OF A REFLECTION IN THE DIRECT SOLUTION OF PLANE ELASTICITY PROBLEMS ON MULTIPLY-CONNECTED DOMAINS*
}

\author{
BY \\ PAUL RAUSCH $\dagger$ \\ Air Force Institute of Technology
}

1. Introduction. Several general methods for finding solutions of plane elasticity problems on simply-connected regions have been presented in the work of N. I. Muskhelishvili [3]. One of these methods, which he calls the method of linear relationship, consists of mapping the material region onto a region which is bounded by a circle and in which the problem can be reduced to a Hilbert problem by means of a reflection across the circular boundary. The mapping of the material region is an essential part of this method as it is presented by Muskhelishvili, and so it appears that it cannot ordinarily be used for problems on multiply-connected regions.

In an attempt to circumvent the need for a mapping of the material region, MilneThomson [1] has suggested a method in which a reflection across the non-circular boundary in the original plane is used to reduce the problem to a Hilbert problem. $\mathrm{He}$ assumes, however, that the reflection of the material region across each of the boundaries is contained in the nonmaterial portion of the original plane.

It is shown in this paper that this assumption is generally invalid because of the multi-valuedness of the reflection functions, but that these functions may be used to reduce the problem to a Hilbert problem if the original plane is replaced by a number of Riemann surfaces. The material region will be treated as having connectivity $n$ in the first part of this paper.

2. The reflection function for an interior boundary. A reflection function**, $z_{i}(\bar{z})$, for an interior boundary $\Gamma_{j}$, is a function which relates some point $z_{i}$ to every point $z$ in the material region $L$, so that if $z$ approaches the point $t$ on $\Gamma_{i}$ from $L, z_{j}$ will approach $t$ from $S_{i}$, the region across $\Gamma_{i}$ from $L$ (Figure 1).

This function may be constructed by using the mapping function

$$
a=\omega_{j}(\zeta)
$$

which transforms the region outside of $\Gamma_{i}$ into the region in the $\zeta$ plane which is outside of the unit circle $\Gamma_{i}^{*}$. (Starred quantities refer to the images in the $\zeta$ plane of the corresponding unstarred quantities of the $z$ plane.) The mapping function is continued analytically into the interior of the unit circle $\Gamma_{i}^{*}$, and a branch of its inverse $\omega_{i}^{-1}(z)$ selected so that $\zeta$ approaches the point $t^{*}$ on $\Gamma_{i}^{*}$ from $L^{*}$ as $z$ approaches the point $t$ on $\Gamma_{i}$ from $L$.

The reflection across $\Gamma_{i}^{*}$ of the point $\zeta$ in $L^{*}$ is given by

$$
\zeta_{j}=1 / \bar{\zeta}
$$

\footnotetext{
*Received April 19, 1965; revised manuscript received December 1, 1965.

**The bar denotes the complex conjugate.

$\nmid$ Now with Aerospace Corporation.
} 


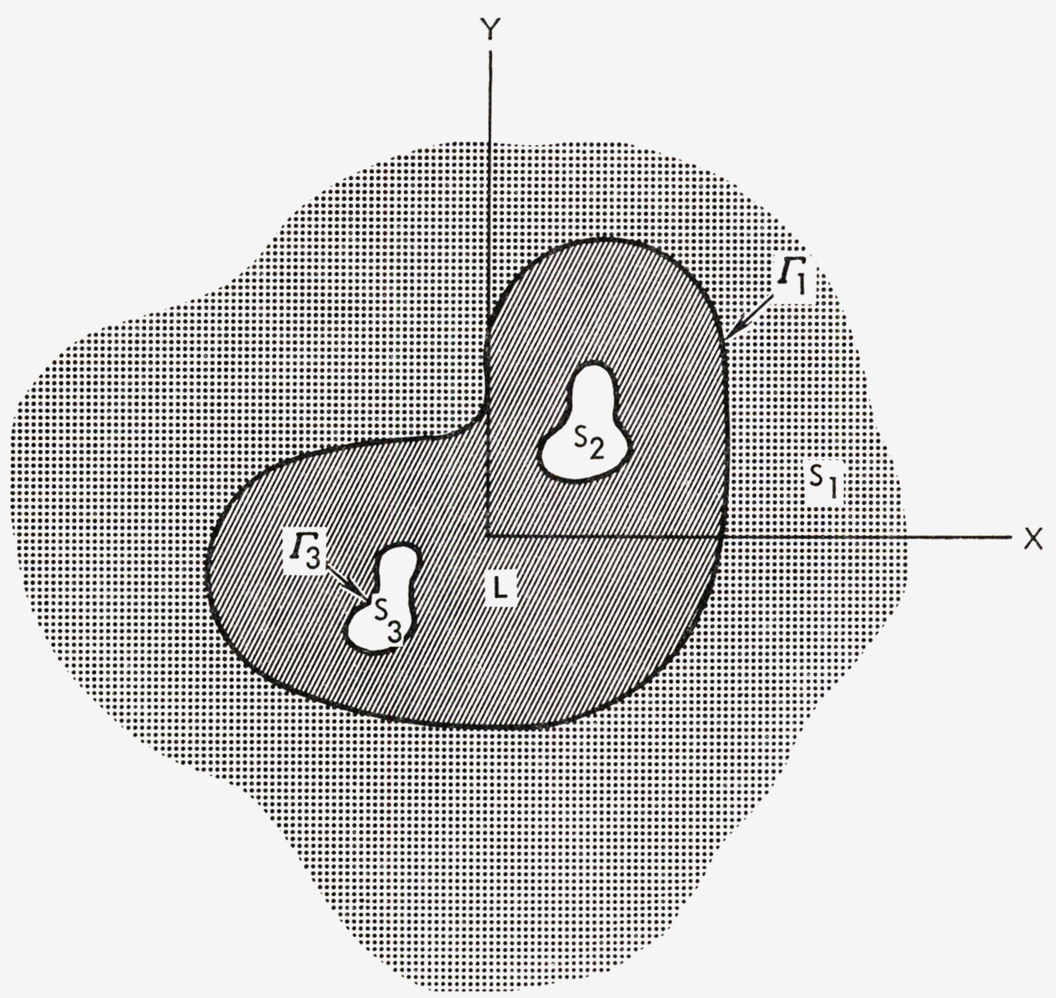

FIG. 1.

This is merely the equation of $\Gamma_{j}^{*}$ but with its domain extended to cover $L^{*}$. Since $z$ is the image of $\zeta$, its reflection across $\Gamma_{j}$ may be taken as the image of the reflection of $\bar{\zeta}$ across $\Gamma_{i}^{*}$. A reflection across $\Gamma_{i}$ is therefore, given by

$$
z_{i}(\bar{z})=\omega_{j}\left[1 / \bar{\omega}_{j}^{-1}(\bar{z})\right] \quad z \text { in } L .
$$

This is merely the equation of $\Gamma_{j}$ but with its domain extended to cover $L$.

The locus of the reflection points across $\Gamma_{j}$ is the region $R_{j}$. Due to the nature of the mapping function (because it generally has a pole at zero) some of the reflection points may be outside of $\Gamma_{j}$, and the region $R_{j}$ may overlap both itself and the region $L$. $R_{i}$ will also contain $(n-1)$ holes due to the other holes in the material region.

While a mapping function has been used in the preceding development of a reflection function, such a function can be found directly from the equation of the boundary unless $\omega_{j}^{\prime}(\zeta)$ is zero on the boundary. An example of how the reflection function can be found from the equation of the boundary is included in the problem which is discussed in the latter part of this paper.

3. Application to the solution of problems on multiply-connected regions. Muskhelishvili [3] gives expressions for the stress and displacement vectors on an arc or contour $C$ in terms of two functions, $\Phi(z)$ and $\Psi(z)$, which are analytic in $L$. Only stress boundary conditions will be considered in this paper; however, the method described may be used in problems with displacement or mixed boundary conditions.

On $C$ the stress vector is given by 


$$
N+i T=\Phi(z)+\bar{\Phi}(\bar{z})-\left[z \bar{\Phi}^{\prime}(\bar{z})+\bar{\Psi}(\bar{z})\right] e^{-2 i \alpha}
$$

where $\alpha$ is the angle between the $x$ axis and the direction normal to $C$. Let us suppose that the equation of the curve $C$ is

$$
z=Z(\bar{z})
$$

It can then be shown that on $C$

$$
e^{-2 i \alpha}=-1 / Z^{\prime}(\bar{z}) .
$$

Thus, equation (3.1) can be rewritten as

$$
N+i T=\Phi(z)+\bar{\Phi}(\bar{z})+\left[z \bar{\Phi}^{\prime}(\bar{z})+\bar{\Psi}(\bar{z})\right] / Z^{\prime}(\bar{z}) \text { on } C .
$$

If $\Phi(z)$ is now continued analytically into $R_{i}$ by means of the expression

$$
\left[\Phi\left(z_{j}\right)+\bar{\Phi}(z)\right] z_{j}^{\prime}(\bar{z})+z_{j}(\bar{z}) \bar{\Phi}^{\prime}(\bar{z})+\bar{\Psi}(\bar{z})=0, \quad z \text { in } L, \quad z_{j} \text { in } R_{j}
$$

then the problem may be formulated solely in terms of $\Phi(z)$, defined in $L$ and $R_{i}$, since $\Psi(z)$ is determinable from $\Phi(z)$ by means of (3.5). The function $\Phi(z)$ shall in this manner be continued into each of the $n$ regions $R_{j}$ in turn, and since this will give $n$ distinct expressions for $\Psi(z)$ (denoted by $\Psi_{i} j=1,2 \cdots n$ ), the condition that all of these expressions for $\Psi(z)$ be identical must be imposed.

If $R_{i}$ overlaps either $L$ or itself, the domain of $\Phi(z)$ must be extended to a Riemann surface $\left(R_{i}+L\right)$ in order that a distinct expression for $\Psi_{i}(z)$ may be obtained for every point in $L$. There will then be a Riemann surface for each reflection function, and these surfaces will generally be composed of a different number of sheets. They must, however, have a common first sheet containing the material region.

For simplicity, our attention is restricted to the case of a doubly-connected material region for which one boundary, $\Gamma_{2}$, requires the use of a Riemann surface of $n$ sheets, and the other, $\Gamma_{1}$, only a plane surface. The expression given in (3.5) may then be used directly for $\Psi_{1}(z)$ with $\Phi(z)$ having a plane domain.

To find an expression for $\Psi_{2}(z)$ which emphasizes the use of the Riemann surfaces, (3.5) must be modified. Let $L_{1}$ be the part of $L$ which is reflected into $S_{2}$, and let $L_{k}(k=1,2,3 \cdots n)$ be the part of $L$ which is reflected onto the $k$ th sheet, $R_{2 k}$, of the Riemann surface. The function $\Phi(z)$ will be denoted by $\Phi(z)$ on the first sheet of the Riemann surface, and by $\Omega_{k}(z)$ on $R_{2 k}$. Equation (3.5) then becomes

$$
\begin{array}{ll}
\Psi_{2}(z)=-\left[\Phi\left(\bar{z}_{2}\right)+\Phi(z)\right] \bar{z}_{2}^{\prime}(z)-\bar{z}_{2}(z) \Phi^{\prime}(z) & z \text { in } L_{1} \\
\Psi_{2}(z)=-\left[\Omega_{k}\left(\bar{z}_{2}\right)+\Phi(z)\right] \bar{z}_{2}^{\prime}(z)-\bar{z}_{2}(z) \Phi^{\prime}(z) & z \text { in } L_{k} .
\end{array}
$$

The problem may now be reduced to a Hilbert problem (see [3] part VI) by replacing the conjugate of $\Psi(z)$ in (3.4) by the conjugate of $\Psi_{2}(z)$ as expressed in equation (3.6), and allowing $z$ to approach $t$ on $\Gamma_{2}$ from $L$ so that $C$ is deformed continuously into $\Gamma_{2}$. This yields the condition

$$
(N+i T)_{\Gamma_{2}}=\Phi^{L}(t)-\Phi^{s_{2}}(t) . \quad t \text { on } \Gamma_{2} .
$$

Use of a similar procedure with (3.4) and (3.5) with $j$ set equal to one yields the condition

$$
(N+i T)_{\Gamma_{2}}=\Phi^{L}(t)-\Phi^{s_{1}}(t) . \quad t \text { on } \Gamma_{1} .
$$


These conditions comprise a Hilbert problem, the solution of which is of the form

$$
\Phi(z)=\frac{1}{2 \pi i} \int_{\Gamma_{2}} \frac{(N+i T)_{\Gamma_{1}} d t}{t-z}+\frac{1}{2 \pi i} \int_{\Gamma_{3}} \frac{(N+i T)_{\Gamma_{2}} d t}{t-z}+P(z) .
$$

The function $P(z)$ must be analytic in $L$, continuous across $\Gamma_{1}$ and $\Gamma_{2}$, cause the displacements to be single valued in $L$, and $\Psi_{1}(z)$ and $\Psi_{2}(z)$ to be analytic in $L$.

4. The problem on a semi-infinite region having an elliptical hole near the boundary. The material region for this problem is shown as cross-hatched in Figure 2. The region is loaded at infinity by a uniform tensile load directed parallel to the straight boundary.

The reflection function for each boundary will be found directly from the equation of the boundary. The equation of $\Gamma_{1}$ is

$$
z+\bar{z}=2 c, \quad z \text { on } \Gamma_{1}
$$

so that

$$
z_{1}(\bar{z})=2 c-\bar{z} .
$$

By using the same procedure as in the appendix, it may be shown that $\Phi(z)$ is analytic in $R_{1} . R_{1}$ will contain a hole as shown in Figure 2.

The equation of $\Gamma_{2}$ is

$$
\left(\frac{z+\bar{z}}{2 a}\right)^{2}-\left(\frac{z-\bar{z}}{2 b}\right)^{2}=1, a^{2}-b^{2}=1,
$$

or

$$
z_{2}(\bar{z})=c_{1} \bar{z} \pm c_{2}\left(\bar{z}^{2}-1\right)^{1 / 2} ; \quad c_{1}=\left(1+2 b^{2}\right) ; \quad c_{2}=2 b\left(1+b^{2}\right)^{1 / 2}
$$

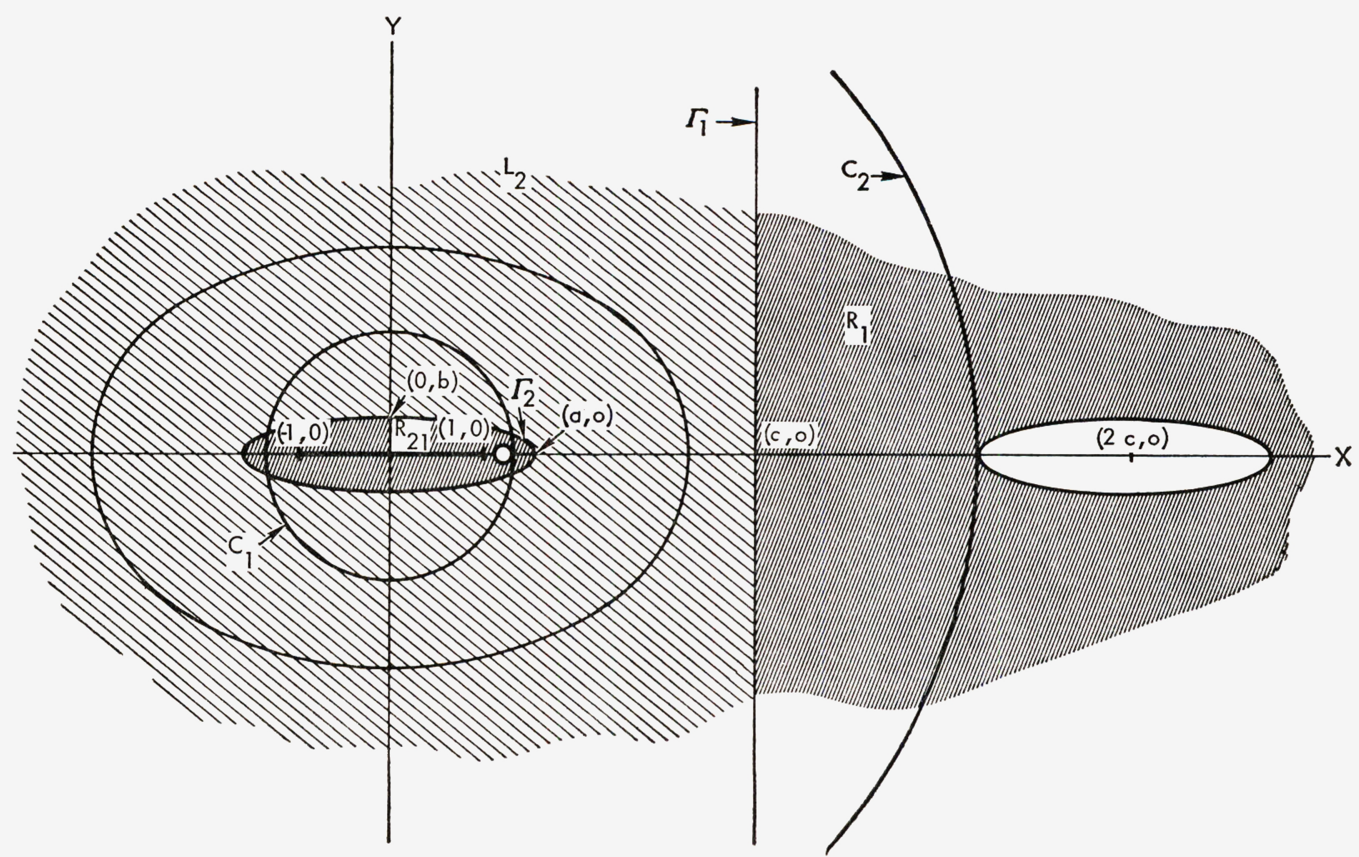


The desired branch of the function $z_{2}(\bar{z})$ may be obtained by letting

$$
z-1=A_{1} e^{i \theta_{1}}, \quad z+1=A_{2} e^{i \theta_{2}} ; \quad-\pi \leq \theta_{1}<\pi, \quad \pi \leq \theta_{2}<3 \pi .
$$

Since the function $z_{2}(\bar{z})$ has the same values at two points in $L, R_{2}$ must be on a Riemann surface of two sheets so that there will be a distinct expression for $\Psi_{2}(z)$ at every point in $L$. The two sheets of this Riemann surface are joined along the part of the real axis for which $-1 \leq x \leq 1$.

The first sheet of the Riemann surface is the plane containing $L$ and $R_{1}$. The part of $R_{2}$ which is in this plane is inside of $\Gamma_{2}$.

$L_{1}$ is the part of $L$ which reflects onto $R_{12}$ and is the region common to $L$ and the interior of the ellipse

$$
\frac{x^{2}}{\left(a^{2}+b^{2}\right)^{2}}+\frac{y^{2}}{4 a^{2} b^{2}}=1
$$

$L_{2}$ is the remainder of $L$.

The singularities of the functions $\Phi(z)$ and $\Omega_{2}(z)$ are discussed in the appendix. For the region under consideration, there are two possible cases:

(1) If $c \leq\left(a^{2}+b^{2}\right)$, the ellipse given in (4.6) will intersect $\Gamma_{1} . R_{21}$ will then contain a hole surrounding the branch point at $(1,0)$, and $R_{22}$ will contain a hole which intersects the branch cut and passes through the point at infinity.

(2) If $c>\left(a^{2}+b^{2}\right)$, the ellipse given in (4.6) will be contained in $L . R_{21}$ will then contain a hole which lies between the point $(a, 0)$ and the branch point at $(1,0) . R_{22}$ will be the entire second sheet. (This case is shown in Figure 2). $\Phi(z)$ and $\Omega_{2}(z)$ will be analytic throughout $R_{2}$ except along the branch cut and at infinity where $\Omega_{2}(z)$ may have a simple pole. The holes mentioned above are not part of $R_{2}$ so they may contain singularities of $\Phi(z)$ and $\Omega_{2}(z)$.

Since both boundaries are stress free, the expression for $\Phi(z)$ given by (3.10) is

$$
\Phi(z)=P(z) .
$$

The function $P(z)$ is analytic throughout $L, R_{1}$, and $R_{21}$ except on the branch cut, and may contain singularities within the holes in $R_{1}$ and $R_{21}$. It may be represented by a Laurent series of the form

$$
P(z)=\sum_{n=-\infty}^{\infty} P_{n} z^{n}
$$

which converges within the annular region bounded by $C_{1}$ and $C_{2}$, as shown in Figure 2.

The condition that $\Psi_{1}(z)$ and $\Psi_{2}(z)$ are identical is used to find the coefficients in the Laurent series for $P(z)$. This condition, which is called the compatibility condition by Milne-Thomson, is

$$
\bar{P}(2 c-z)+P(z)-(2 c-z) P^{\prime}(z)=-\left[\bar{P}\left(\bar{z}_{2}\right)+P(z)\right] \bar{z}_{2}(z)-z_{2}(z) P^{\prime}(z) .
$$

The coefficients $P_{n}$ may be found from this condition by solving the set of equations obtained by setting to zero the coefficient of each power of $z$. All of the terms in (4.9) must converge in some subregion of $L$. If $\Psi_{1}(z)$ and $\Psi_{2}(z)$ are equal in this subregion, they are equal throughout $L$, since they are analytic everywhere in $L$.

For the first term on each side of the equation to converge in a subregion, $P(z)$ must converge in the reflection of that subregion across each boundary. Such a subregion 
exists in a neighborhood of the part of the real axis between $\Gamma_{1}$ and $\Gamma_{2}$. The expression found for $P(z)$ from (4.9) is the desired expression for $\Phi(z)$ in this neighborhood. This neighborhood is the part of $L$ in which the stresses are the largest, and so it is the region of greatest interest. $\Phi(z)$ may be found in other parts of the plane by analytic continuation, and $\Omega_{2}(z)$ may be found from the condition that $\Psi(z)$ must be continuous across the boundary between $L_{1}$ and $L_{2}$.

5. Conclusions. The final solution of the problem discussed in section four is tedious because of the form of (4.9). Other problems will be further complicated by the difficulty in finding the equations of other boundaries in the form of (4.1) and (4.3). It is believed, however, that problems on regions bounded by non-conic curves and of connectivity higher than two may be solved, by methods similar to those presented in this paper, with the aid of a digital computer.

APPENDIX: The Singularities of $\Phi\left(z_{2}\right)$ aNd $\Omega_{k}\left(z_{2}\right)$

From (3.6) and (3.7), $\Phi\left(z_{2}\right)$ and $\Omega_{k}\left(z_{2}\right)$ may be expressed as

$$
\begin{aligned}
& \Phi\left(z_{2}\right)=-\bar{\Phi}(\bar{z})-\left[z_{2}(\bar{z}) \bar{\Phi}^{\prime}(\bar{z})+\bar{\Psi}_{2}(\bar{z})\right] / z_{2}^{\prime}(\bar{z}), \quad z \text { in } L_{1} \\
& \Omega_{k}\left(z_{2}\right)=-\bar{\Phi}(\bar{z})-\left[z_{2}(\bar{z}) \bar{\Phi}^{\prime}(\bar{z})+\bar{\Psi}_{2}(\bar{z})\right] / z_{2}(\bar{z}) . \quad z \text { in } L_{k}
\end{aligned}
$$

Changing variables from $z$ to $\zeta$ by means of the mapping function, allows these expressions to be rewritten as

$$
\begin{aligned}
& \Phi^{*}\left(\zeta_{2}\right)=-\Phi^{*}\left(1 / \zeta_{2}\right)+\left[\omega_{2}\left(\zeta_{2}\right) \Phi^{*^{\prime}}\left(1 / \zeta_{2}\right)+\bar{\omega}_{2}^{\prime}\left(1 / \zeta_{2}\right) \bar{\Psi}\left(1 / \zeta_{2}\right)\right] / \zeta_{2}^{2} \omega_{2}^{\prime}\left(\zeta_{2}\right), \\
& \Omega^{*}\left(\zeta_{2}\right)=-\bar{\Phi}^{*}\left(1 / \zeta_{2}\right)+\left[\omega_{2}\left(\zeta_{2}\right) \Phi^{*^{\prime}}\left(1 / \zeta_{2}\right)+\bar{\omega}_{2}^{\prime}\left(1 / \zeta_{2}\right) \bar{\Psi}\left(1 / \zeta_{2}\right)\right] / \zeta_{2} \omega_{2}^{\prime}\left(\zeta_{2}\right),
\end{aligned}
$$

where

$$
\Phi^{*}(\zeta)=\Phi\left[\omega_{2}(\zeta)\right], \quad \Omega_{k}^{*}(\zeta)=\Omega_{k}\left[\omega_{2}(\zeta)\right], \quad \Psi^{*}(\zeta)=\zeta\left[\omega_{2}(\zeta)\right] .
$$

Since $\Phi(z)$ and $\Psi(z)$ are analytic in $L$ and $\omega_{2}(\zeta)$ is analytic throughout the $\zeta$ plane except at zero and infinity, $\Phi^{*}(\zeta)$ and $\Psi^{*}(\zeta)$ are analytic in $L^{*}$ except at infinity (if it is in $\left.L^{*}\right)$, and $\Phi^{*}\left(1 / \zeta_{2}\right)$ and $\Psi^{*}\left(1 / \zeta_{2}\right)$ are analytic in $R_{2}^{*}$ except at zero. $\omega_{2}^{\prime}\left(1 / \zeta_{2}\right)$ is also analytic in $R_{2}^{*}$ except at zero. $\omega_{2}^{\prime}\left(\zeta_{2}\right)$ has zeros at the critical points of $\omega_{2}(\zeta)$ which correspond to the branch points of $\bar{z}_{2}(z)$ in $R_{2}$. Therefore, both $\Phi^{*}\left(\zeta_{2}\right)$ and $\Omega_{k}^{*}\left(\zeta_{2}\right)$ are analytic in $R_{2}^{*}$ except at zero and the critical points.

The inverse of the mapping function is now used to return to the $z$ plane. The inverse function is analytic in $R_{2}$ except at infinity and the branch points of $\bar{z}_{2}(z)$ where it has branch points. Thus, $\Phi\left(z_{2}\right)$ is analytic on its domain except at the branch points, and $\Omega_{k}(z)$ is analytic on its domain except at the branch points and infinity.

Examination of (A.2) and (A.3) and the form of the mapping function reveals that $\Omega_{k}\left(z_{2}\right)$ has a simple pole at infinity and both functions go to infinity as $1 / z$ at the branch points.

\section{REFERENCES}

[1] L. M. Milne-Thomson, Plane elastic systems, Springer-Verlag, Berlin, Göttingen, Heidelberg, 1960

[2] T. P. Mitchell and W. E. Warren, On the method of Milne-Thomson in plane elasticity, Journal of Applied Mechanics, (December 1962)

[3] N. I. Muskhelishvili, Some basic problems of the mathematical theory of elasticity, Third, revised and augmented edition, Noordhoff., Groningen, Holland, 1953

[4] H. B. Wilson, Jr., Stresses due to rotary inertia forces in a hollow circular cylinder, Presented at the First Southeastern Conference on Theoretical and Applied Mechanics, 1962 